\title{
8-Aminoguanosine Exerts Diuretic, Natriuretic, and Glucosuric Activity via Conversion to 8-Aminoguanine, Yet Has Direct Antikaliuretic Effects
}

\author{
Edwin K. Jackson and Zaichuan Mi \\ Department of Pharmacology and Chemical Biology, University of Pittsburgh School of Medicine, Pittsburgh, Pennsylvania
}

Received July 1, 2017; accepted September 18, 2017

\begin{abstract}
8-Aminoguanosine induces diuresis, natriuresis, glucosuria, and antikaliuresis. These effects could be mediated via 8-aminoguanosine's metabolism to 8-aminoguanine. In this study, we tested this hypothesis in anesthetized rats. First, we demonstrated that at 55- to 85-minutes post-i.v. administration, 8-aminoguanosine and 8-aminoguanine $(33.5 \mu \mathrm{mol} / \mathrm{kg})$ significantly increased urine volume [ml/30 $\mathrm{min}: 8$-aminoguanosine from $0.3 \pm 0.1$ to $0.9 \pm 0.1$ (mean \pm S.E.M.; $n=7$ ); 8-aminoguanine from $0.3 \pm 0.1$ to $1.5 \pm 0.2$ $(n=8)$, sodium excretion $(\mu \mathrm{mol} / 30 \mathrm{~min}: 8$-aminoguanosine from $12 \pm 5$ to $109 \pm 21$; 8-aminoguanine from $18 \pm 8$ to $216 \pm 31$ ), and glucose excretion $(\mu \mathrm{g} / 30 \mathrm{~min}$ : 8 -aminoguanosine from $18 \pm 3$ to $159 \pm 41$; 8-aminoguanine from $17 \pm 3$ to $298 \pm 65$ ). Both compounds significantly decreased potassium excretion ( $\mu \mathrm{mol} / 30 \mathrm{~min}$ : 8-aminoguanosine from $62 \pm 7$ to $39 \pm 9$; 8-aminoguanine from $61 \pm 10$ to $34 \pm 6$ ). Next, we administered 8-aminoguanosine and 8-aminoguanine i.v. $(33.5 \mu \mathrm{mol} / \mathrm{kg})$ and measured renal interstitial (microdialysis probes) 8-aminoguanosine
\end{abstract}

and 8-aminoguanine. The i.v. administration of 8-aminoguanosine and 8-aminoguanine similarly increased renal medullary interstitial levels of 8-aminoguanine [nanograms per milliliter; 8-aminoguanosine from $4 \pm 1$ to $1025 \pm 393(n=6)$, and 8-aminoguanine from $2 \pm 1$ to $1069 \pm 407(n=6)]$. Finally, we determine the diuretic, natriuretic, glucosuric, and antikaliuretic effects of intrarenal artery infusions of 8-aminoguanosine and 8-aminoguanine $(0.1,0.3$, and $1 \mu \mathrm{mol} / \mathrm{kg} / \mathrm{min})$. 8-Aminoguanine increased urine volume and sodium and glucose excretion by the ipsilateral kidney, yet had only mild effects at the highest dose in the contralateral kidney. Intrarenal infusions of 8-aminoguanosine did not induce diuresis, natriuresis, or glucosuria in either the ipsilateral or contralateral kidney, yet decreased potassium excretion in the ipsilateral kidney. Together these data confirm that the diuretic, natriuretic, and glucosuric effects of 8-aminoguanosine are not direct, but require metabolism to 8-aminoguanine. However, 8-aminoguanosine has direct antikaliuretic effects.

\section{Introduction}

Endogenous guanosine and guanine can be modified in the 8 position to yield a number of endogenous 8-substituted guanosines and guanines including 8-nitroguanosine (Akaike et al., 2003), 8-aminoguanosine (Sodum et al., 1993), 8-hydroxyguanosine (Park et al., 1992), 8-nitroguanine (Ohshima et al., 2006), 8-hydroxyguanine (Fraga et al., 1990), and 8-hydroxy-2'deoxyguanosine (Lam et al., 2012). Until recently, nothing was known concerning the effects of these naturally-occurring 8 -substituted guanosine and guanine compounds on the cardiovascular system or kidneys.

To address this knowledge gap, we systematically examined a series of 8-substituted guanosines and guanines on the cardiovascular system and kidneys in rats (Jackson et al., 2016). In particular, we examined the renal effects in vivo of i.v. administration of guanosine, guanine, 8-nitroguanosine, 8-nitroguanine, 8-hydroxyguanosine, 8-hydroxyguanine, 8-hydroxy2 '-deoxyguanosine, 8-aminoguanosine, and 8-aminoguanine.

This work was supported by the National Institutes of Health [Grants DK091190, HL069846, DK068575, HL109002, and DK079307].

https://doi.org/10.1124/jpet.117.243758.
We observed that 8-aminoguanosine and 8-aminoguanine produced marked diuresis, natriuresis, and glucosuria, and reduced potassium excretion (Jackson et al., 2016). Moreover, long-term oral treatment of rats with 8-aminoguanosine and 8 -aminoguanine attenuated the development of deoxycorticosterone/salt-induced hypertension (Jackson et al., 2016).

In the systemic circulation, exogenous 8-aminoguanosine can be converted to 8 -aminoguanine by purine nucleoside phosphorylase (PNPase) (Osborne and Barton, 1986). Therefore, it is conceivable that 8-aminoguanosine does not act directly on the kidneys to induce the aforementioned effects but rather functions as a prodrug (if exogenous) or a prohormone (if endogenous). The goal of the present study was to test this hypothesis.

\section{Materials and Methods}

Materials. 8-Aminoguanosine and 8-aminoguanine were purchased from Toronto Research Chemicals (Toronto, Ontario, Canada).

Animals. This study employed male Sprague-Dawley rats (Charles River, Wilmington, MA) that were approximately 16 weeks of age. The Institutional Animal Care and Use Committee approved all procedures. The investigation conforms to National Institutes of Health Guide for the Care and Use of Laboratory Animals.

ABBREVIATIONS: GFR, glomerular filtration rate; HR, heart rate; LC-MS/MS, liquid chromatography-tandem mass spectrometry; MABP, mean arterial blood pressure; PE, polyethylene; PNPase, purine nucleoside phosphorylase; RBF, renal blood flow. 
Protocol 1. After inducing anesthesia with Inactin $(90 \mathrm{mg} / \mathrm{kg}$, i.p.), each rat was positioned on an isothermal pad. A thermometer probe was inserted into the rectum, and the rat's body temperature was continuously monitored and maintained at $37^{\circ} \mathrm{C}$ by adjusting the distance of a heat lamp from the animal's body. Using a polyethylene (PE)-240 tubing, the trachea was cannulated to aid respiration. Next, a PE-50 cannula was inserted into the carotid artery and connected to a digital blood pressure analyzer (Micro-Med, Louisville, KY) for continuous measurement of mean arterial blood pressure (MABP) and heart rate (HR). A PE-50 cannula was placed in the jugular vein, and an infusion of $0.9 \%$ saline $(50 \mu \mathrm{l} / \mathrm{min})$ was begun. Finally, a PE-10 cannula was inserted into the left ureter for timed collections of urine. After a stabilization period of approximately 1 hour, urine was collected for 30 minutes, whereas MABP and HR were time averaged (period 1: 0-30 minutes into protocol). Next, 8-aminoguanosine (33.5 $\mu \mathrm{mol} / \mathrm{kg} ; 1 \mathrm{ml} / \mathrm{kg}$ ) or 8 -aminoguanine $(33.5 \mu \mathrm{mol} / \mathrm{kg} ; 1 \mathrm{ml} / \mathrm{kg})$ or vehicle $(0.9 \%$ saline containing $0.03 \mathrm{~N} \mathrm{HCl} ; 1 \mathrm{ml} / \mathrm{kg})$ was administered i.v. as a bolus. The dose was selected based on our previous work (Jackson et al., 2016). Rats were randomized to treatment groups, and rats in each group received only one treatment. Ten minutes after the treatments, urine was collected for another 30 minutes (period 2: 40-70 minutes into protocol). After a 15-minute rest period, urine was again collected for 30 minutes (period 3: 85-115 minutes into protocol). During each urine collection period, MABP and HR were continuously recorded. Also, at the midpoint of each 30-minute urine collection period, a blood sample $(0.5 \mathrm{ml})$ was obtained for measurement of plasma creatinine (Creatinine Colorimetric Assay Kit; catalog number 500701; Cayman Chemical, Ann Arbor, MI) and aldosterone (aldosterone enzyme-linked immunosorbent assay kit; catalog number, ADI900-173; Enzo Life Sciences, Farmingdale, NY) and for determination of sodium and potassium by flame photometry (Model IL-943; Instrumentations Laboratory, Lexington, MA). Sodium and potassium were also measured in urine (flame photometry), as was glucose (Glucose Colorimetric Assay Kit; catalog number 10009582; Cayman Chemical). Creatinine clearance was calculated as an estimate of glomerular filtration rate (GFR).

Protocol 2. Rats were prepared in a similar fashion to that described above for protocol 1, but with modifications. Specifically, microdialysis probes were inserted into the cortex and medulla of the right kidney and were perfused at $2 \mu \mathrm{l} / \mathrm{min}$ with $0.9 \%$ saline. The microdialysis probes used in this protocol were from Bioanalytical Systems (West Lafayette, IN; CMA/20 microdialysis probe $4 \mathrm{~mm}$; outer diameter of $0.5 \mathrm{~mm}$; 20,000-Da membrane cutoff). After initiating the probe perfusions, the infusion rate of $0.9 \%$ saline via the jugular vein was reduced to $25 \mu \mathrm{l} / \mathrm{min}$ to avoid volume overload. At this point in the protocol, the rats were allowed to stabilize for 2 hours in order for the kidney to recover from the trauma induced by the probe insertions.

Next, microdialysate from the renal cortex and medulla and urine were collected for 30 minutes, and MABP and HR were time averaged during this first (basal) period. Rats then were randomized to receive 8 -aminoguanosine or 8-aminoguanine or vehicle at the dose and manner described above for protocol 1 . Ten minutes after treatments, microdialysate from the renal cortex and medulla and urine were again collected for 30 minutes, and MABP and HR were time averaged during this treatment period. After 15 minutes, this procedure was repeated one more time. 8-Aminoguanosine and 8-aminoguanine were measured in urine and microdialysate using ultra-performance liquid chromatography-tandem mass spectrometry (LC-MS/MS), as described below.

Protocol 3. Rats were prepared in a similar fashion to that described above for protocol 1, but with a few additions. In this regard, PE-10 cannulas were inserted into both the left and right ureters for urine collection from both kidneys simultaneously. Also, a 1-mm transit-time flow probe was positioned on left renal artery and connected to a flowmeter (model T-206; Transonic Systems, Ithaca, NY) for continuous measurement of renal blood flow (RBF). Since placing a flow probe on left renal artery partially denervates the kidney, to ensure that both kidneys were under similar conditions, both kidneys were denervated. Finally, a 30 -gauge needle was inserted into the lumen of the left renal artery, and an infusion of $0.9 \%$ saline at $50 \mu \mathrm{l} / \mathrm{min}$ was initiated. The infusion rate of $0.9 \%$ saline via the jugular vein was reduced to $25 \mu \mathrm{l} / \mathrm{min}$ to avoid volume overload.

After a stabilization period of 1 hour, urine was collected from both kidneys for 15 minutes (for urine volume, sodium, potassium, and glucose), and MABP and HR were time averaged during this period. Also, RBF was recorded at 5,10 , and 15 minutes into the 15-minute urine collection period and averaged. Next, rats received an infusion into the left renal artery of either 8-aminoguanine or 8 -aminoguanosine $(0.1 \mu \mathrm{mol} / \mathrm{kg} / \mathrm{min}$ at $50 \mu \mathrm{l} / \mathrm{min})$ or vehicle (also $50 \mu \mathrm{l} / \mathrm{min}$ ). Ten minutes later, urine was again collected for 15 minutes and all measurements were repeated. In rats receiving 8-aminoguanine or 8-aminoguanosine, the dose of these compounds was increased to $0.3 \mu \mathrm{mol} / \mathrm{kg} / \mathrm{min}$. After 10 minutes, urine was again collected for 15 minutes, and all measurements were repeated. The procedure was repeated once more after increasing the dose of 8-aminoguanosine or 8 -aminoguanine to $1 \mu \mathrm{mol} / \mathrm{kg} / \mathrm{min}$. Sodium, potassium, and glucose were measured in all urine samples, as described above for protocol 1.

Assay for 8-Aminoguanosine and 8-Aminoguanine. Purines in urine and microdialysate were measured by LC-MS/MS using a method similar to that previously described by us (Jackson et al., 2009), but with modifications. In this regard, purines were separated by reversed-phase ultra-performance liquid chromatography (UPLC BEH C18 column, 1.7- $\mu$ m beads; $2.1 \times 150 \mathrm{~mm}$; Waters, Milford, MA) and quantified by selected reaction monitoring using a triplequadrupole mass spectrometer (TSQ Quantum-Ultra; ThermoFisher Scientific, San Jose, CA) with a heated electrospray ionization source. The mobile phase was a linear gradient flow rate $(300 \mu \mathrm{l} / \mathrm{min})$ of $1 \%$ acetic acid in water (pH, 3; mobile phase $\mathrm{A}$ ) and $100 \%$ methanol (mobile phase B), and was delivered with a Waters Acquity ultra-performance liquid chromatographic system. The gradient $(\mathrm{A} / \mathrm{B})$ settings were as follows: from 0 to 2 minutes, $99.6 \% / 0.4 \%$; from 2 to 3 minutes, to $98.0 \%$ $2.0 \%$; from 3 to 4 minutes, to $85.0 \% / 15.0 \%$; from 4 to 6.5 minutes, to $99.6 \% / 0.4 \%$. The instrument parameters were as follows: sample tray temperature, $10^{\circ} \mathrm{C}$; column temperature, $50^{\circ} \mathrm{C}$; ion spray voltage, $4.0 \mathrm{kv}$; ion transfer tube temperature, $350^{\circ} \mathrm{C}$; source vaporization temperature, $320^{\circ} \mathrm{C}$; Q2 CID gas, argon at $1.5 \mathrm{mTorr}$; sheath gas, nitrogen at $60 \mathrm{psi}$; auxillary gas, nitrogen at $35 \mathrm{psi}$; Q1/Q3 width, 0.7/0.7 U full-width half-maximum; scan width, 0.6 U; scan time, 0.01 seconds. For 8-aminoguanosine, the parent ion was $\mathrm{m} / \mathrm{z}=299$, the daughter ion was $\mathrm{m} / \mathrm{z}=167$, and the retention time was 3.64 minutes. For 8 -aminoguanine, the parent ion was $\mathrm{m} / \mathrm{z}=167$, the daughter ion was $\mathrm{m} / \mathrm{z}=150$, and the retention time was 1.50 minutes. The internal standard for 8-aminoguanosine was ${ }^{13} \mathrm{C}_{10},{ }^{15} \mathrm{~N}_{5}$-guanosine (parent ion, $\mathrm{m} / \mathrm{z}=299$; daughter ion, $\mathrm{m} / \mathrm{z}=162$; retention time, 3.10 minutes). The internal standard for 8 -aminoguanine was ${ }^{13} \mathrm{C}_{2},{ }^{15} \mathrm{~N}$ guanine (parent ion, $\mathrm{m} / \mathrm{z}=155$; daughter ion, $\mathrm{m} / \mathrm{z}=138$; retention time, 1.56 minutes)

Statistics. Statistical analysis was performed using one- or twofactor repeated-measures analysis of variance, followed by Fisher's least significant difference test if the effects in the analysis of variance were significant. The criterion of significance was $P<0.05$. Values are presented as mean \pm S.E.M.

\section{Results}

Protocol 1. To confirm the dosing paradigm, we examined in a head-to-head study (with randomization of treatment groups) the effects of an i.v. bolus of vehicle (control group), 8-aminoguanosine $(33.5 \mu \mathrm{mol} / \mathrm{kg})$, and 8 -aminoguanine $(33.5 \mu \mathrm{mol} / \mathrm{kg})$ in anesthetized rats. As shown in Figs. 1 and 2, respectively, neither 8-aminoguanosine nor 8-aminoguanine acutely affected MABP (Figs. 1A and 2A), HR (Figs. 1B and 2B), or plasma aldosterone 

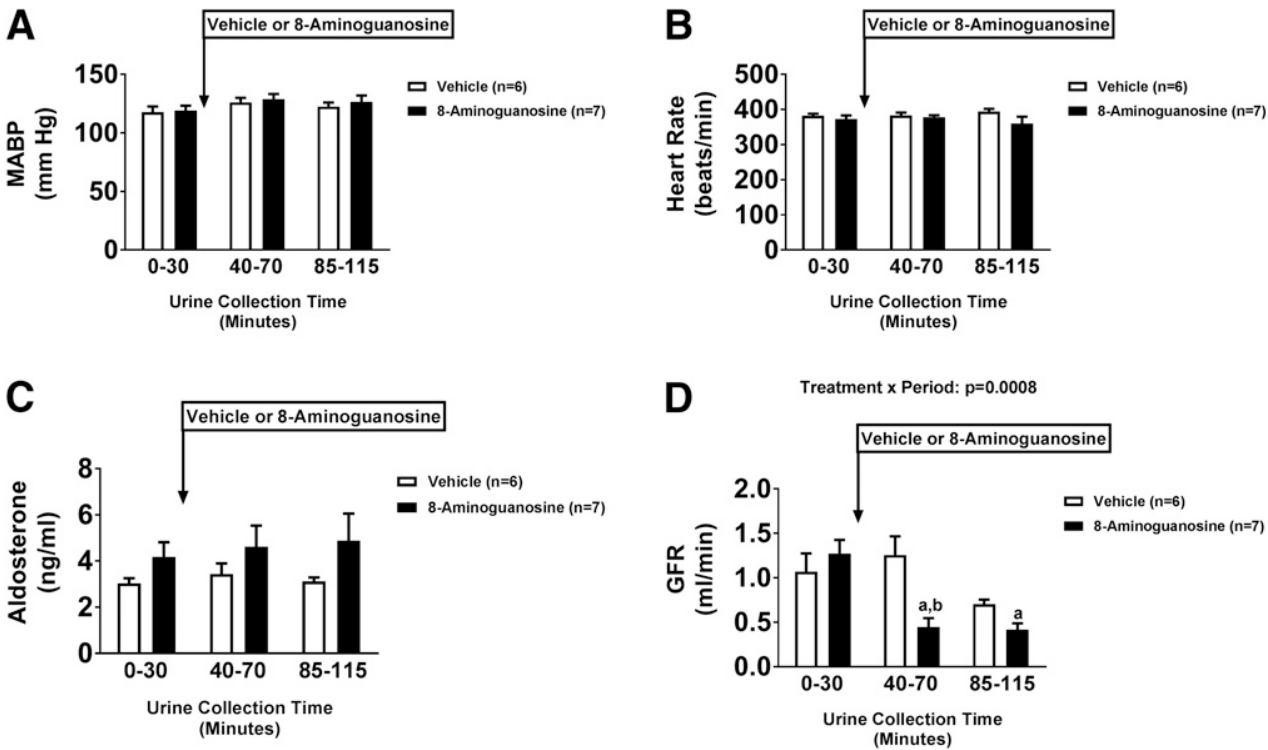

D Treatment $x$ Period: $\mathrm{p}=0.0008$
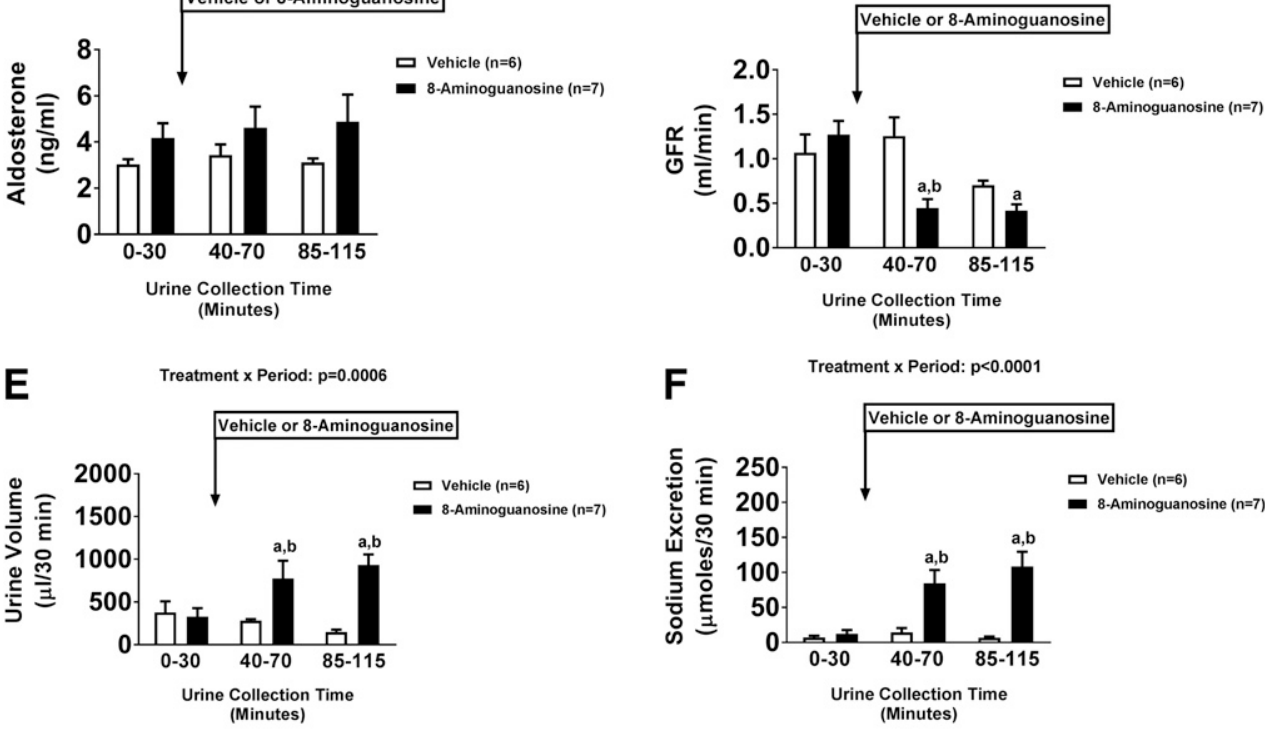

Fig. 1. Bar graphs depict (A) MABP, (B) heart rate, (C) plasma concentration of aldosterone, (D) GFR (estimated by creatinine clearance), (E) urine volume, (F) urinary sodium excretion, $(\mathrm{G})$ urinary potassium excretion, and $(\mathrm{H})$ urinary glucose excretion in rats administered

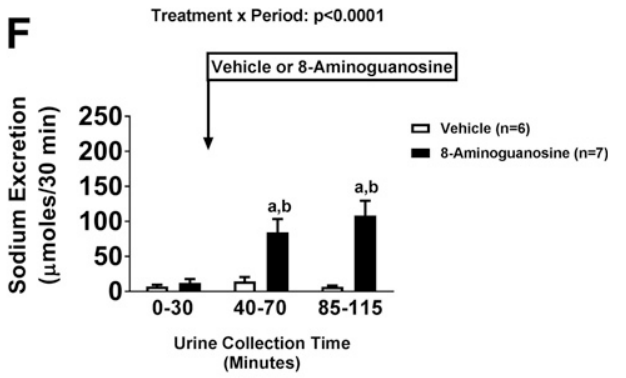
either 8-aminoguanosine $(33.5 \mu \mathrm{mol} / \mathrm{kg}$; $1 \mathrm{ml} / \mathrm{kg})$ or its vehicle $(1 \mathrm{ml} / \mathrm{kg})$ i.v. The $P$ value given for treatment $\times$ period is the interaction term in a repeated-measures, two-factor analysis of variance. ${ }^{\text {aSignifi- }}$ cantly different from corresponding 0-30 minute period. ${ }^{b}$ Significantly different from corresponding vehicle period. Values are means and S.E.M.
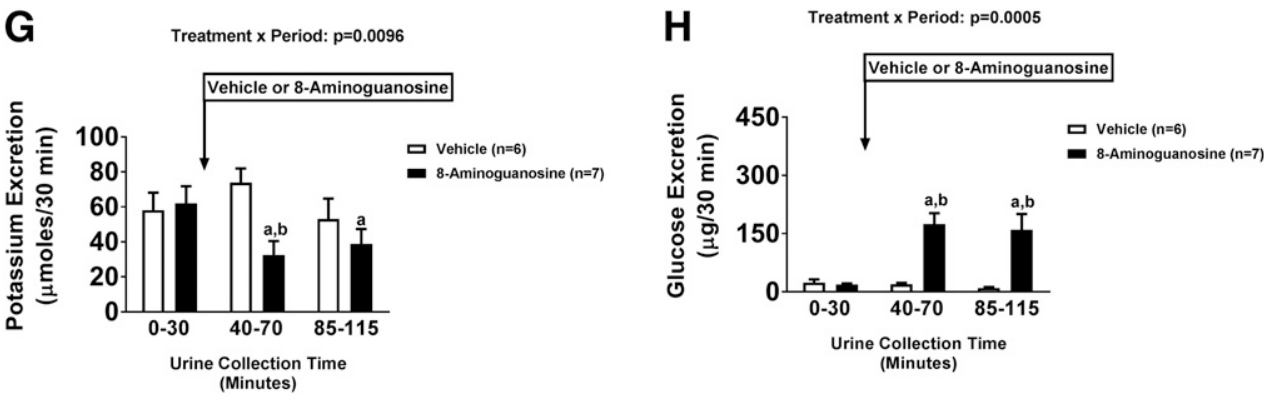

concentrations (Figs. 1C and 2C). However, both compounds significantly decreased GFR (Figs. 1D and 2D) and urinary potassium excretion (Figs. $1 \mathrm{G}$ and $2 \mathrm{G}$ ). Also, both compounds significantly increased urine volume (Figs. $1 \mathrm{E}$ and $2 \mathrm{E}$ ), urinary sodium excretion (Figs. $1 \mathrm{~F}$ and $2 \mathrm{~F}$ ), and urinary glucose excretion (Figs. $1 \mathrm{H}$ and $2 \mathrm{H}$ ). These effects of i.v. administered 8-aminoguanine and 8-aminoguanosine were qualitatively the same and quantitatively similar. Therefore, we chose the dosing paradigm established in this protocol for protocol 2.

Protocol 2. In this protocol, we inserted microdialysis probes into the renal cortex and medulla of anesthetized rats, let them stabilize for 2 hours, and then administered an i.v. bolus of vehicle (control group), 8-aminoguanosine (33.5 $\mu \mathrm{mol} / \mathrm{kg})$, or 8 -aminoguanine $(33.5 \mu \mathrm{mol} / \mathrm{kg})$ and measured using LC-MS/MS the levels of these compounds in the renal cortex and medulla and in the urine. As shown in Fig. 3, administration of 8-aminoguanine and 8-aminoguanosine caused immediate, sustained, similar, and large increases in the interstitial levels of 8-aminoguanine in the renal cortex (Fig. 3A) and renal medulla (Fig. 3B). Vehicle per se had no effect. In contrast, neither 8-aminoguanine nor 8-aminoguanosine affected cortical (Fig. 3C) or medullary (Fig. 3D) interstitial levels of 8-aminoguanosine. In the urine (Fig. 4), administration of 8-aminoguanine and 8-aminoguanosine also caused immediate, sustained, similar, and large increases in 8-aminoguanine (Fig. 4A). 8-Aminoguanine did not affect urinary levels of 8-aminoguanosine (Fig. 4B); although 8-aminoguanosine did cause an increase in urinary 8-aminoguanosine levels, this response was variable and short-lived (Fig. 4B).

Protocol 3. Next, we infused increasing doses $(0,0.1,0.3$, and $1.0 \mu \mathrm{mol} / \mathrm{kg} / \mathrm{min}$ ) of 8-aminoguanosine and 8-aminoguanine into the left renal artery of anesthetized rats while monitoring MABP and RBF. We also measured urine volume and urinary excretion of sodium, potassium, and glucose from both the infused (treated; ipsilateral) and noninfused (within 

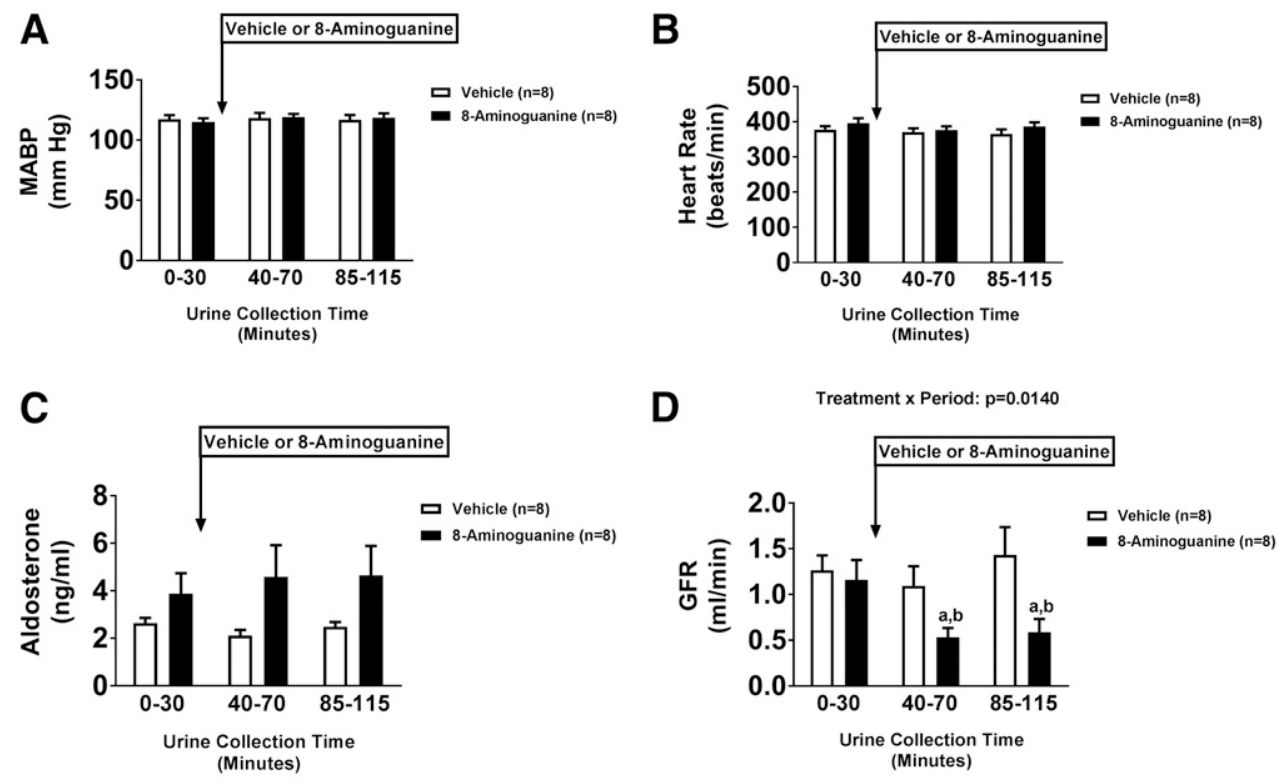

Fig. 2. Bar graphs depict (A) MABP, (B) heart rate, (C) plasma concentration of aldosterone, (D) GFR (estimated by creatinine clearance), (E) urine volume, (F) urinary sodium excretion, (G) urinary potassium excretion, and $(\mathrm{H})$ urinary glucose excretion in rats administered either 8 -aminoguanine $(33.5 \mu \mathrm{mol} / \mathrm{kg}$; $1 \mathrm{ml} / \mathrm{kg})$ or its vehicle $(1 \mathrm{ml} / \mathrm{kg})$ i.v. The $P$ value given for treatment $\times$ period is the interaction term in a repeated-measures, two-factor analysis of variance. ${ }^{a}$ Significantly different from corresponding 0 - to 30 -minute period. ${ }^{\text {b Significantly }}$ different from corresponding vehicle pe-

riod. Values are means and S.E.M.
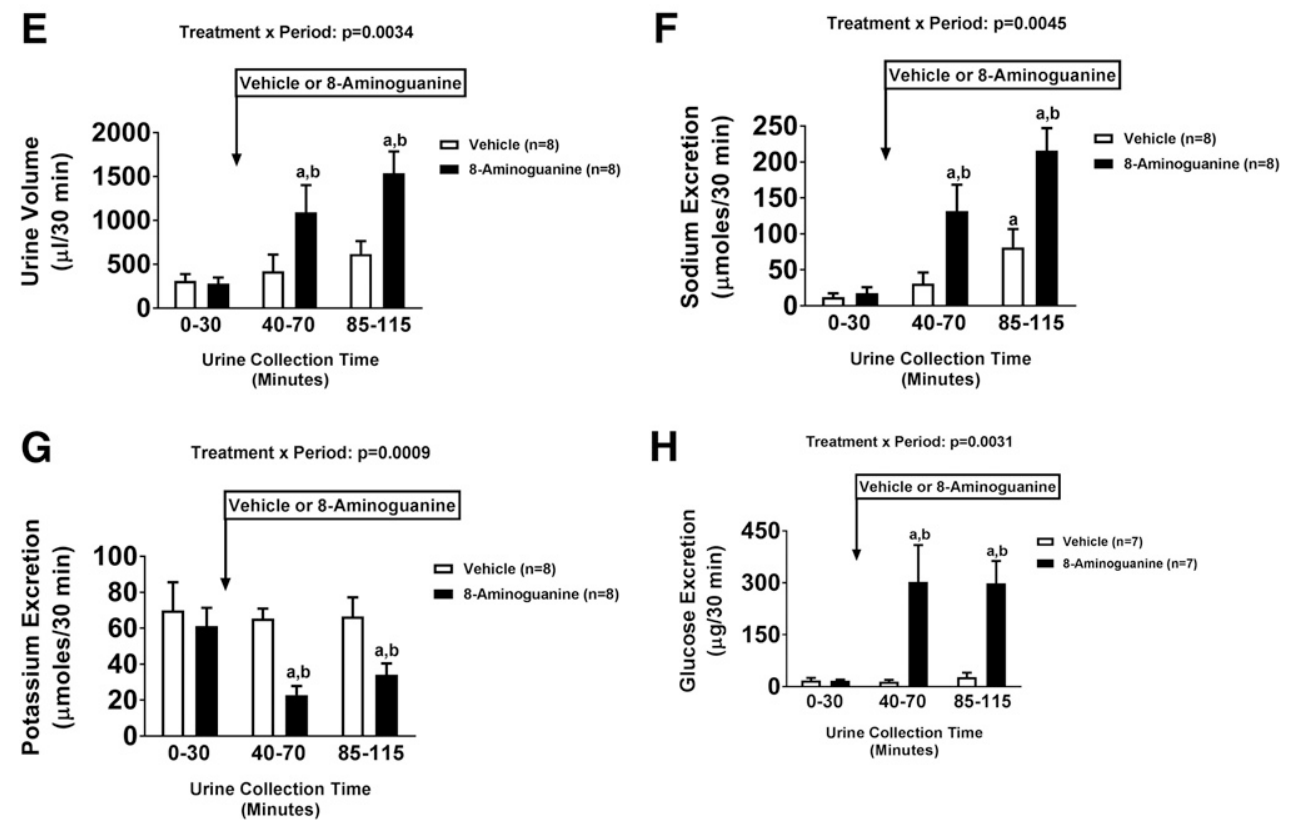

animal control; contralateral) kidneys. A control group in which only vehicle was infused into the left kidney was also included.

As illustrated in Fig. 5, intrarenal artery infusions of vehicle, 8-aminoguanine, and 8-aminoguanosine did not affect MABP (Fig. 5A) or RBF (Fig. 5B). In the control group that received intrarenal artery infusions of vehicle (Fig. 6), there were no significant differences between the ipsilateral versus the contralateral kidneys at the same period with regard to either urine volume (Fig. 6A) or urinary excretion of sodium (Fig. 6B), potassium (Fig. 6C), or glucose (Fig. 6D).

As with the control group, in the group that received an intrarenal artery infusion of 8-aminoguanosine (Fig. 7), there were no significant differences between the ipsilateral versus the contralateral kidneys at the same time period with regard to either urine volume (Fig. 7A) or urinary excretion of sodium (Fig. 7B) or glucose (Fig. 7D). However, at $0.1,0.3$, and $1 \mu \mathrm{mol} / \mathrm{kg} / \mathrm{min}$ infusions of 8 -aminoguanosine, the urinary excretion of potassium by the ipsilateral kidney was significantly lower compared with that by the contralateral kidney at the corresponding time period (Fig. 7C).

In the group receiving intrarenal administration of 8 -aminoguanine (Fig. 8), at $0.1,0.3$, and $1 \mu \mathrm{mol} / \mathrm{kg} / \mathrm{min}$ infusions of 8 -aminoguanine, the urine (Fig. $8 \mathrm{~A}$ ), sodium (Fig. 8B), and glucose (Fig. 8D) excretions by the ipsilateral kidney were significantly higher compared with those of the contralateral kidney at the corresponding time period. 8 -Aminoguanine at 0.1 and $0.3 \mu \mathrm{mol} / \mathrm{kg} / \mathrm{min}$ had no effect on the excretion of urine, sodium, or glucose by the contralateral kidney; yet compared with the basal level, 8-aminoguanine at $1 \mu \mathrm{mol} / \mathrm{kg} / \mathrm{min}$ slightly increased the excretion of urine, sodium, and glucose by the contralateral kidney. However, urinary potassium excretion (Fig. 8C) was not significantly 

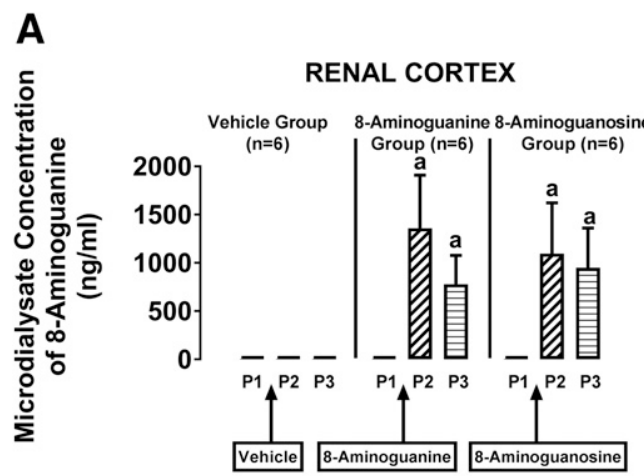

C

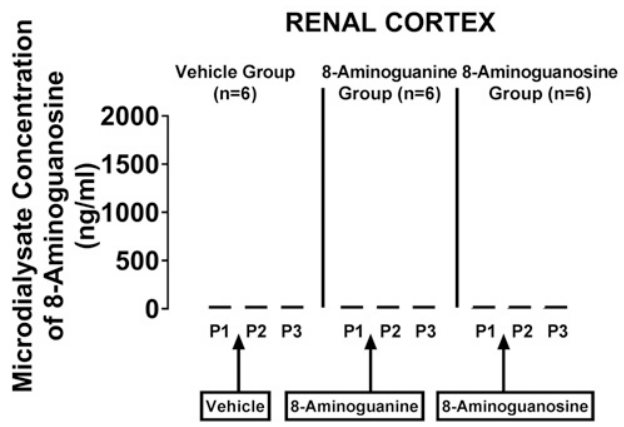

B

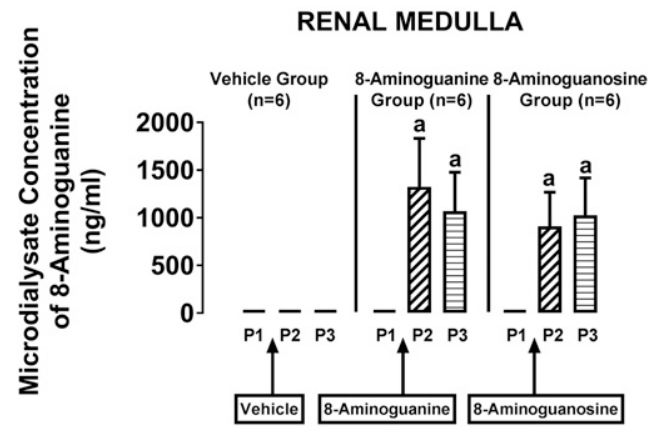

D

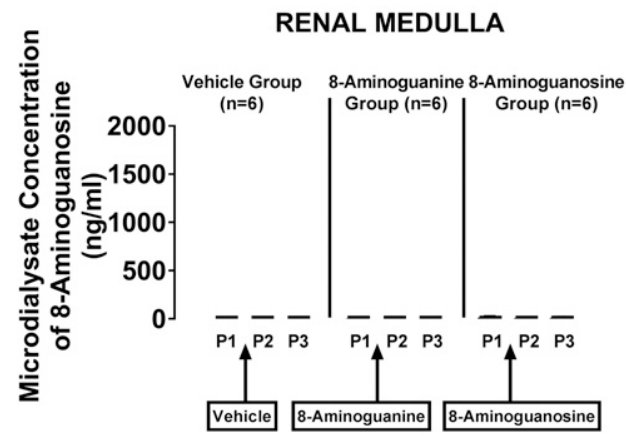

Fig. 3. Bar graphs depict the concentrations of 8-aminoguanine (A and $\mathrm{B}$ ) and 8-aminoguanosine $(\mathrm{C}$ and $\mathrm{D})$ in the microdialysate obtained from the renal cortex (A and $\mathrm{C})$ and renal medulla (B and $\mathrm{D})$ before treatments $(\mathrm{P} 1)$ and after i.v. administration (P2 and P3) of either vehicle (1 ml/kg),

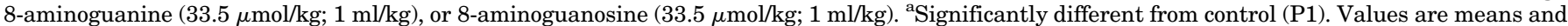
S.E.M.

different between the ipsilateral and contralateral kidneys at any dose of 8-aminoguanine.

\section{Discussion}

Guanosine and guanine derivatives in which the 8 position of the purine ring is modified exist in vivo. Examples include 8-nitroguanosine (Akaike et al., 2003), 8-aminoguanosine (Sodum et al., 1993), 8-hydroxyguanosine (Park et al., 1992), 8-nitroguanine (Ohshima et al., 2006), 8-hydroxyguanine (Fraga et al., 1990), and 8-hydroxy-2'-deoxyguanosine (Lam et al., 2012). Because the cardiovascular and renal effects of these compounds had not been reported, recently we systematically examined in vivo the cardiovascular and renal effects of several naturally-occurring 8-substituted guanosine and guanine derivatives (Jackson et al., 2016). In this regard, we observed that i.v. administration of 8-nitroguanine, 8-hydroxyguanosine, 8-hydroxyguanine, 8-aminoguanosine, and 8-aminoguanine caused diuresis and natriuresis; and, of these compounds, 8-aminoguanosine and 8-aminoguanine were particularly efficacious (Jackson et al., 2016). Both markedly increased urine volume and increased the urinary excretion of sodium and glucose, while suppressing urinary potassium excretion (Jackson et al., 2016). Because of the unique qualities of 8-aminoguanosine and 8-aminoguanine, we are currently investigating more fully the pharmacology of these two compounds.

Because exogenous 8-aminoguanosine can be converted to 8-aminoguanine by purine nucleoside phosphorylase (Osborne and Barton, 1986), we entertained the hypothesis that 8 -aminoguanosine might be a prodrug or prohormone (depending upon whether the source of the 8 -aminoguanosine is exogenous or endogenous). That is to say, we considered the concept that the effects of 8-aminoguanosine on the kidneys are not direct but are mediated by its metabolism to 8-aminoguanine.

To address this hypothesis, we first compared head-to-head the renal effects of i.v. administered 8-aminoguanosine and 8 -aminoguanine. Both compounds increased urine volume and the urinary excretion of sodium and glucose, while decreasing the urinary excretion of potassium. Until now, we had not examined the effects of i.v. 8-aminoguanosine or 8 -aminoguanine on plasma aldosterone or GFR. Neither compound significantly affected plasma aldosterone, which rules out any diuretic/natriuretic activity mediated by inhibition of aldosterone production. Of note, both compounds decreased GFR. This suggests that: 1) the diuretic/natriuretic/glucosuric effects of 8-aminoguanosine and 8-aminoguanine are not mediated by increasing filtered load of electrolytes or glucose, and 2) possibly the increased sodium excretion caused by these compounds activates tubuloglomerular feedback to reduce GFR. The most important aspect of this experiment, however, is the finding that both compounds behave qualitatively the same and quantitatively similar when administered i.v., a finding that is consistent with, but not proof of, the hypothesis that 8 -aminoguanosine is an 8-aminoguanine prodrug/prohormone.

If 8-aminoguanosine is truly a prodrug/prohormone of 8-aminoguanine, then systemic administration of pharmacologically 
A

\section{Urine}

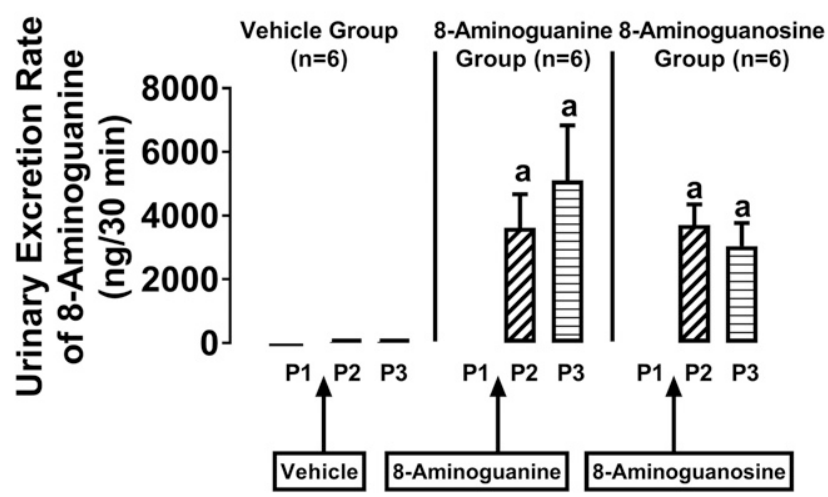

B
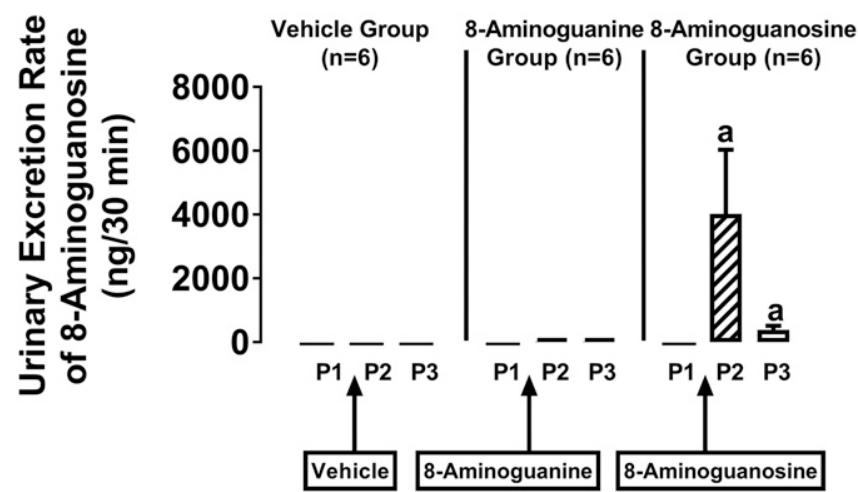

Fig. 4. Bar graphs depict the concentrations of 8 -aminoguanine (A) and 8-aminoguanosine (B) in the urine before treatments (P1) and after i.v. administration (P2 and P3) of either vehicle $(1 \mathrm{ml} / \mathrm{kg})$, 8-aminoguanine (33.5 $\mu \mathrm{mol} / \mathrm{kg} ; 1 \mathrm{ml} / \mathrm{kg})$, or 8 -aminoguanosine $(33.5 \mu \mathrm{mol} / \mathrm{kg} ; 1 \mathrm{ml} / \mathrm{kg})$. a Significantly different from control (P1). Values are means and S.E.M.

active doses of 8-aminoguanosine should produce intrarenal levels of 8-aminoguanine that are achieved by matched doses of 8-aminoguanine that also have similar pharmacological activity. To test this prediction, we measured intrarenal levels of 8-aminoguanosine and 8-aminoguanine by inserting microdialysis probes into the renal cortex and medulla. Next, we injected i.v. a dose of 8-aminoguanosine and matching dose of 8-aminoguanine that we confirmed in our first protocol produced qualitatively the same and quantitatively similar renal responses. Importantly, i.v. administration of 8-aminoguanosine increased renal interstitial levels of 8-aminoguanine to the same degree as did matched i.v. doses of 8-aminoguanine. Moreover, 8-aminoguanosine did not increase renal interstitial levels of 8-aminoguanosine. This confirms that 8-aminoguanosine is an 8-aminoguanine prodrug (if exogenous) or prohormone (if endogenous). This hypothesis is further corroborated by the finding that i.v. 8-aminoguanosine and 8-aminoguanine caused similar increases in urinary levels of 8-aminoguanine. Although i.v. 8-aminoguanosine did increase urinary levels of 8-aminoguanosine, this effect was quite variable and limited in time.

To further explore our hypothesis, we next administered low doses of 8-aminoguanosine and 8-aminoguanine directly into the left renal artery while monitoring the excretion of urine,

\section{A Mean Arterial Blood Pressure}

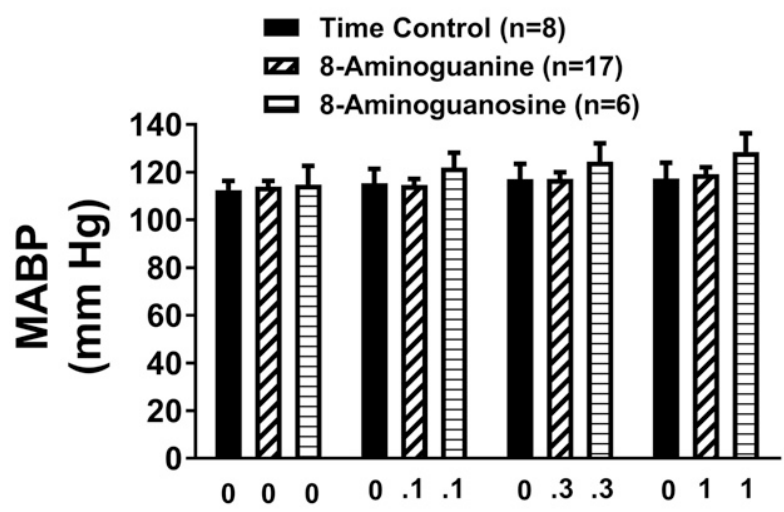
Intrarenal Artery Infusion Rate of 8-Aminoguanine or 8-Aminoguanosine ( $\mu \mathrm{mol} / \mathrm{kg} / \mathrm{min})$

\section{Renal Blood Flow}

B

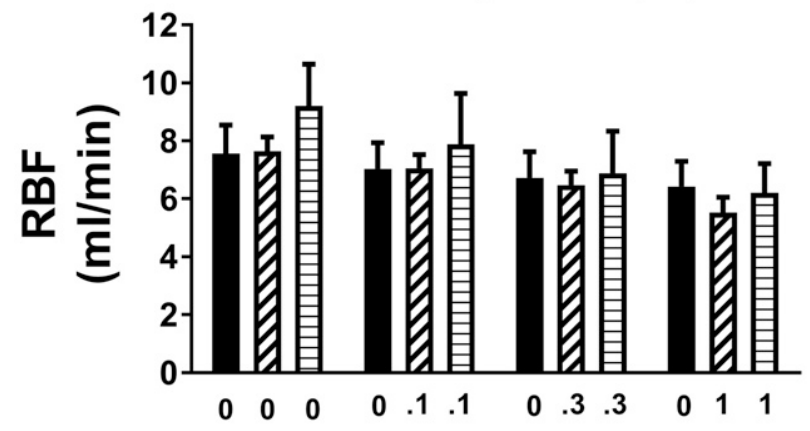

Intrarenal Artery Infusion Rate of 8-Aminoguanine or 8-Aminoguanosine ( $\mu \mathrm{mol} / \mathrm{kg} / \mathrm{min})$

Fig. 5. Bar graphs depict the (A) MABP and (B) renal blood flow in rats receiving intrarenal artery infusions (left kidney) of either vehicle, 8 -aminoguanine $(0.1,0.3$, and $1 \mu \mathrm{mol} / \mathrm{kg} / \mathrm{min})$, or 8 -aminoguanosine $(0.1$, 0.3 , and $1 \mu \mathrm{mol} / \mathrm{kg} / \mathrm{min}$ ). Values are means and S.E.M.

sodium, potassium, and glucose from both the ipsilateral (treated) and contralateral (control) kidneys. Our logic was that if 8-aminoguanosine is an 8-aminoguanine prodrug and if the conversion of arterial 8-aminoguanosine to 8-aminoguanine occurs largely in the systemic circulation, then administration of 8-aminoguanosine into the renal artery should be inactive, whereas administration of matched doses of 8-aminoguanine should induce diuresis and natriuresis.

Consistent with our hypothesis, intrarenal artery infusions of 8-aminoguanosine did not affect urine volume or the urinary output of sodium or glucose at any of the doses tested. In contrast, at all doses tested, administration of 8-aminoguanine into the renal artery markedly increased urine excretion, sodium excretion, and glucose excretion in the 
A

\section{Effects of Time/Vehicle on Urine Volume}

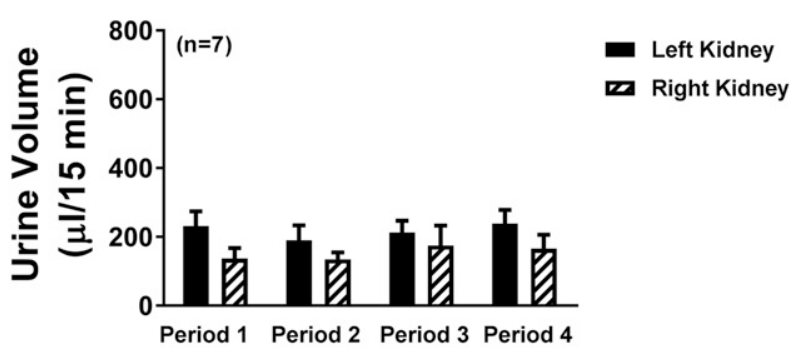

C Effects of Time/Vehicle on Potassium Excretion

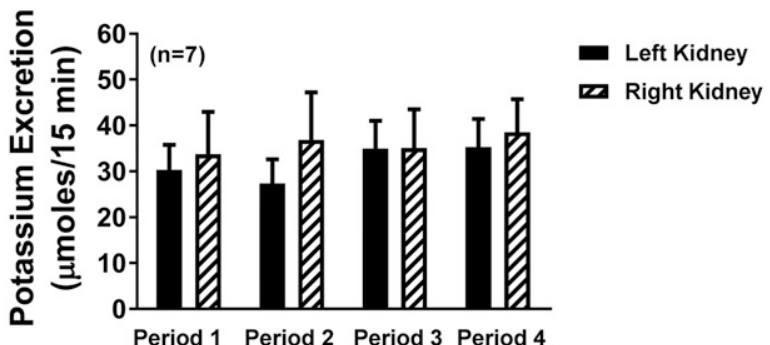

B

Effects of Time/Veichle on Sodium Excretion

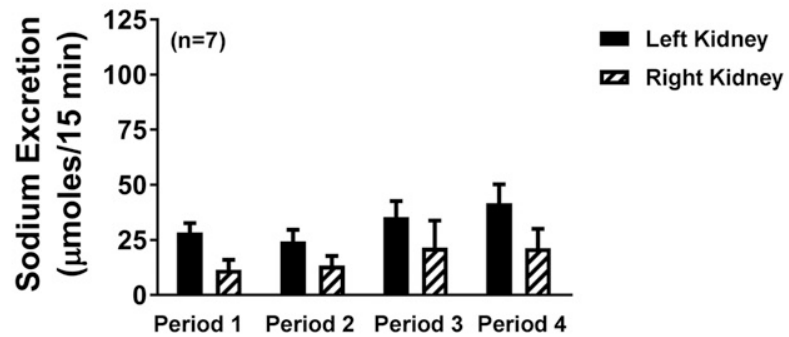

D

Effects of Time/Vehicle on Glucose Excretion

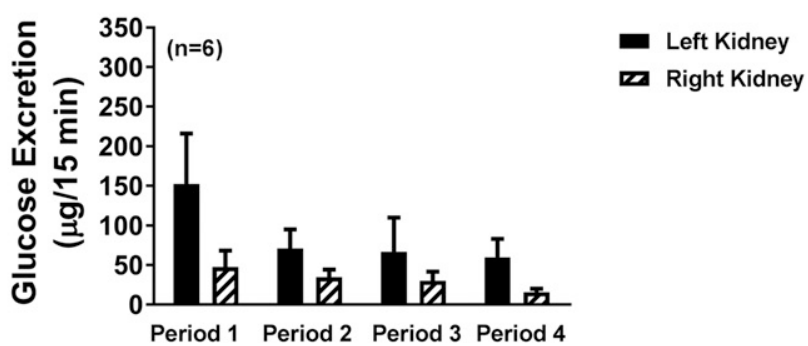

Fig. 6. Bar graphs depict the (A) urine excretion, (B) urinary sodium excretion, (C) urinary potassium excretion, and (D) urinary glucose excretion by the left and right kidneys during direct infusions into the left renal artery of the vehicle for 8-aminoguanosine and 8-aminoguanine during four 15-minute periods. Values are means and S.E.M.

infused kidney. Yet, in the noninfused kidney, only the highest dose of 8-aminoguanine affected these parameters, and even then only slightly. These results are highly consistent with the concept that 8-aminoguanine has direct intrarenal actions to cause diuresis, natriuresis, and glucosuria, whereas 8 -aminoguanosine does not and requires metabolism to 8-aminoguanine.

Interestingly, 8-aminoguanine decreased potassium excretion when administered i.v., but not when administered directly into the renal artery. This is likely related to the fact that the intrarenal doses of 8-aminoguanine had to be low because of poor solubility. We observed that higher concentrations of 8-aminoguanine precipitated and clogged the needle inserted into the renal artery; so low doses were used in these experiments. At the doses employed, most likely the increased presentation of sodium to the distal nephron offset the tendency for 8-aminoguanine to decrease potassium excretion such that the net effect of intrarenal 8-aminoguanine was potassium sparing, but did not decrease potassium excretion overall.

A very important finding is that, despite the fact that intrarenal artery administration of 8-aminoguanosine did not affect urine volume or sodium or glucose excretion, intrarenal artery infusions of 8-aminoguanosine did significantly reduce potassium excretion by the ipsilateral kidney. This suggests that 8-aminoguanosine directly induces antikaliuresis and that this aspect of the pharmacology of 8-aminoguanosine does not require conversion to 8-aminoguanine. Unlike 8-aminoguanine, intrarenal artery infusions of 8-aminoguanosine did not increase sodium excretion, and therefore the antikaliuretic effects of 8-aminoguanosine were observable (i.e., were not masked by increased sodium delivery to the distal nephron). Taken together, these findings suggest two very distinct mechanisms of action of 8-aminoguanine/8-aminoguanosine. One mechanism causes diuresis, natriuresis, and glucosuria and is mediated directly by 8-aminoguanine and indirectly by 8-aminoguanosine; and a separate mechanism causes antikaliuresis and is mediated directly by both 8 -aminoguanine and 8-aminoguanosine. This hypothesis must be further tested by experiments designed to elucidate the molecular mechanisms underlying the effects of 8-aminoguanine and 8-aminoguanosine on the renal nephron.

Both 8-aminoguanosine and 8-aminoguanine inhibit PNPase. However, 8-aminoguanine is at least 10 -fold more potent than 8-aminoguanosine in this regard (Kazmers et al., 1981; Gilbertsen and Dong, 1985). Therefore, the fact that 8 -aminoguanosine is an 8-aminoguanine prodrug is consistent with the hypothesis that the diuretic/natriuretic/glucosuric activity of these compounds is related to 8-aminoguanine-induced inhibition of renal PNPase. We are currently testing this hypothesis.

The present study provides strong evidence that the diuretic, natriuretic, and glucosuric activity of 8-aminoguanosine is due to its conversion to 8-aminoguanine. Because intrarenal artery administration of 8-aminoguanosine does not induce diuresis/ natriuresis/glucosuria, whereas i.v. administration does, our results suggest that arterial 8-aminoguanosine is processed to 8 -aminoguanine mostly in the systemic circulation. It is important to point out that this does not preclude the possibility that endogenous 8-aminoguanosine produced within some 
A

Effects of 8-Aminoguanosine on Urine Volume

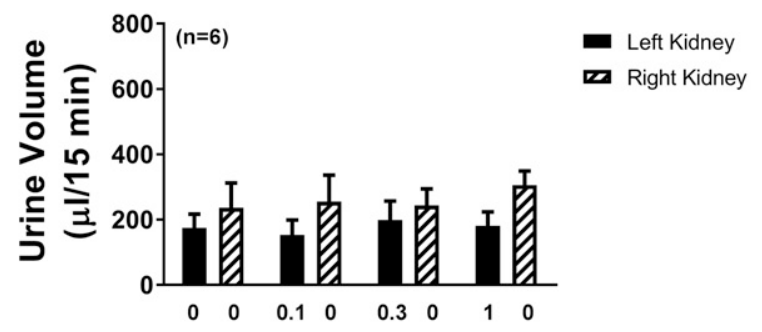

Intrarenal Artery Infusion Rate of 8-Aminoguanosine $(\mu \mathrm{mol} / \mathrm{kg} / \mathrm{min})$

\section{C}

Effects of 8-Aminoguanosine on Potassium Excretion

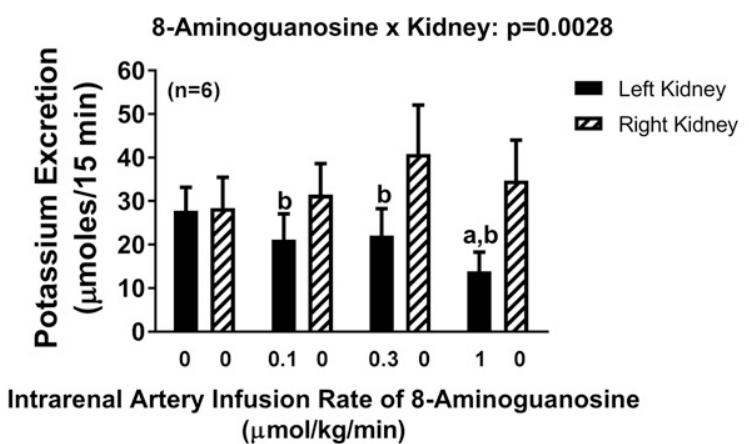

B

Effects of 8-Aminoguanosine on Sodium Excretion

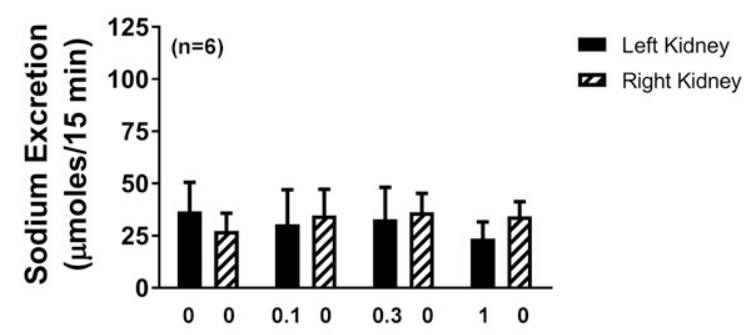

Intrarenal Artery Infusion Rate of 8-Aminoguanosine $(\mu \mathrm{mol} / \mathrm{kg} / \mathrm{min})$
Effects of 8-Aminoguanosine on Glucose Excretion

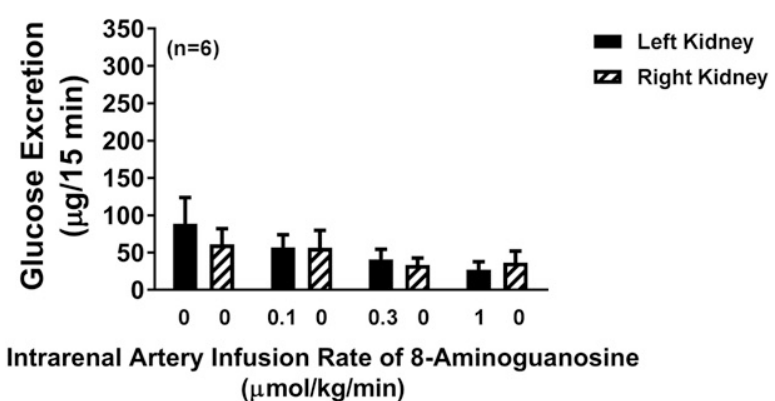

Fig. 7. Bar graphs depict the (A) urine excretion, (B) urinary sodium excretion, (C) urinary potassium excretion, or (D) urinary glucose excretion by the left and right kidneys during direct infusions into the left renal artery of increasing doses of 8 -aminoguanosine $(0.1,0.3$, and $1 \mu \mathrm{mol} / \mathrm{kg} / \mathrm{min}) \mathrm{during}$ four 15-minute periods. The $P$ value given for treatment $\times$ period is the interaction term in a repeated-measures, two-factor analysis of variance.

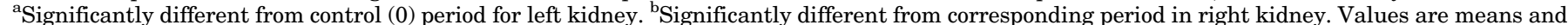
S.E.M.

nonvascular compartments within the kidney may be metabolized to 8-aminoguanine locally in the kidney and thus exert effects on nephron function. Additional experiments are required to test this hypothesis.

Probably the metabolism of 8-aminoguanosine to 8aminoguanine occurs mostly in the liver and red blood cells because of the following: 1) both hepatocytes (Brass and Mody, 1995) and red blood cells (Back et al., 1992) are richly endowed with PNPase, and 2) hepatocytes and red blood cells represent a large mass of cells. The metabolism of 8-aminoguanosine to 8-aminoguanine must be rapid because: 1 ) the time course of the diuretic/natriuretic/glucosuric effects of 8-aminoguanosine and 8-aminoguanine is similar, 2) 8-aminoguanosine produces renal interstitial levels of 8 -aminoguanine with a similar time course to that of 8-aminoguanine, and 3) the urinary levels of 8-aminoguanosine drop from approximately $4 \mu \mathrm{g} / 30 \mathrm{~min}$ to $0.4 \mu \mathrm{g} / 30 \mathrm{~min}$ in 45 minutes. However, there is currently no precise information on the plasma pharmacokinetics of 8-aminoguanosine or whether 8-aminoguanine undergoes further metabolism.

An important issue is the clinical relevance of 8aminoguanosine and 8-aminoguanine. Previously we published that both compounds attenuated the development of hypertension in uninephrectomized rats treated with deoxycorticosterone and provided $1 \% \mathrm{NaCl}$ as drinking water
(Jackson et al., 2016). In unpublished, preliminary experiments, we have observed antihypertensive effects of these compounds in Dahl SS rats on a high-salt diet, in ZDSD rats with the metabolic syndrome, and in spontaneously hypertensive rats. We have also observed that both 8-aminoguanosine and 8-aminoguanine extended the life span of Dahl SS rats on a high-salt diet, apparently by preventing strokes. In ZDSD rats, we found that 8-aminoguanine decreased HbA1C levels. Finally, we have evidence of beneficial effects of these compounds in pulmonary hypertension and sickle cell disease. Although these studies are ongoing, we believe that 8-aminoguanosine and/or 8-aminoguanine may have significant utility in the management of renal and cardiovascular diseases.

In conclusion, the present study does the following: 1) confirms the diuretic, natriuretic, glucosuric, and antikaliuretic effects of systemically administered 8-aminoguanosine and 8-aminoguanine; 2) shows that the renal effects of these compounds are not due to inhibition of aldosterone release; 3) demonstrates that with respect to inducing diuresis, natriuresis, and glucosuria, 8-aminoguanosine is an 8-aminoguanine prodrug; and 4) suggests that the mechanism by which 8 -aminoguanosine and 8-aminoguanine cause antikaliuresis is distinct from the mechanism by which they induce diuresis, natriuresis, and glucosuria. Further studies 
A

Effects of 8-Aminoguanine on Urine Volume

8-Aminoguanine $x$ Kidney: $p=0.0005$

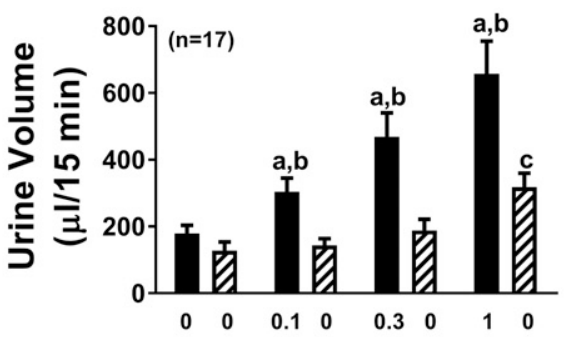

Intrarenal Artery Infusion Rate of 8-Aminoguanine ( $\mu \mathrm{mol} / \mathrm{kg} / \mathrm{min}$ )

C

\section{Effects of 8-Aminoguanine on Potassium Excretion}
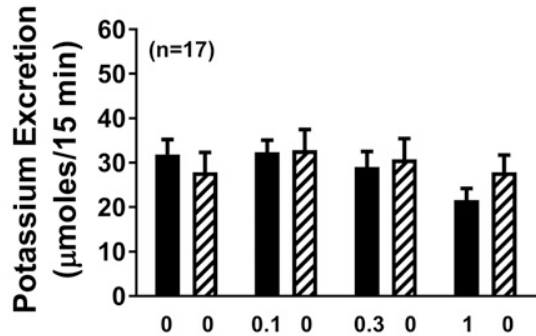

- Left Kidney

might Kidney

Intrarenal Artery Infusion Rate of 8-Aminoguanine $(\mu \mathrm{mol} / \mathrm{kg} / \mathrm{min})$
B

Effects of 8-Aminoguanine on Sodium Excretion

8-Aminoguanine $x$ Kidney: $p=0.0004$

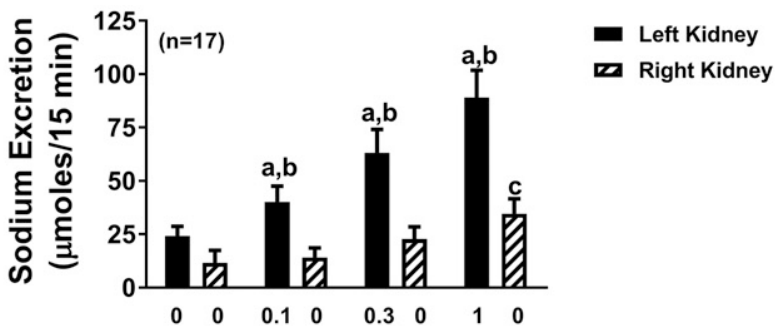

Intrarenal Artery Infusion Rate of 8-Aminoguanine ( $\mu \mathrm{mol} / \mathrm{kg} / \mathrm{min}$ )

\section{Effects of 8-Aminoguanine on Glucose Excretion}

8-Aminoguanine $x$ Kidney: $p=0.0123$

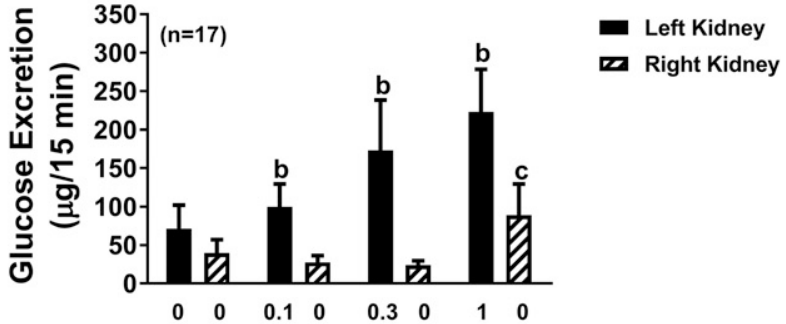

Intrarenal Artery Infusion Rate of 8-Aminoguanine ( $\mu \mathrm{mol} / \mathrm{kg} / \mathrm{min}$ )

Fig. 8. Bar graphs depict the (A) urine excretion, (B) urinary sodium excretion, (C) urinary potassium excretion, or (D) urinary glucose excretion from the left and right kidneys during direct infusions into the left renal artery of increasing doses of 8 -aminoguanine $(0.1,0.3$, and $1 \mu \mathrm{mol} / \mathrm{kg} / \mathrm{min}) \mathrm{during}$ four 15 -minute periods. The $P$ value given for treatment $\times$ period is the interaction term in a repeated-measures, two-factor analysis of variance. ${ }^{a}$ Significantly different from control (0) period for left kidney. ${ }^{b}$ Significantly different from corresponding period in right kidney. ${ }^{\mathrm{c}}$ Significantly different from control (0) period for right kidney. Values are means and S.E.M.

are warranted to investigate the pharmacology and clinical utility of 8-aminoguanosine and 8-aminoguanine and to explore the physiologic significance of endogenous 8-aminoguanosine and 8-aminoguanine.

\section{Authorship Contributions}

Participated in research design: Jackson.

Conducted experiments: Mi.

Performed data analysis: Jackson.

Wrote or contributed to the writing of the manuscript: Jackson.

\section{References}

Akaike T, Okamoto S, Sawa T, Yoshitake J, Tamura F, Ichimori K, Miyazaki K, Sasamoto K, and Maeda H (2003) 8-Nitroguanosine formation in viral pneumonia and its implication for pathogenesis. Proc Natl Acad Sci USA 100:685-690.

Back DJ, Ormesher S, Tjia JF and Macleod R (1992) Metabolism of $2^{\prime}, 3^{\prime}-$ dideoxyinosine (ddI) in human blood. Br J Clin Pharmacol 33:319-322.

Brass CA and Mody MG (1995) Evaluation of purine nucleoside phosphorylase release as a measure of hepatic endothelial cell injury. Hepatology 21:174-179.

Fraga CG, Shigenaga MK, Park JW, Degan P, and Ames BN (1990) Oxidative damage to DNA during aging: 8-hydroxy-2'-deoxyguanosine in rat organ DNA and urine. Proc Natl Acad Sci USA 87:4533-4537.

Gilbertsen RB and Dong MK (1985) Effects of 8-aminoguanosine, an inhibitor of purine nucleoside phosphorylase, on plasma nucleosides in Wistar rats. Ann N Y Acad Sci 451:313-314.
Jackson EK, Gillespie DG, and Mi Z (2016) 8-Aminoguanosine and 8-aminoguanine exert diuretic, natriuretic, glucosuric, and antihypertensive activity. J Pharmacol Exp Ther 359:420-435.

Jackson EK, Ren J, and Mi Z (2009) Extracellular 2', $3^{\prime}$-cAMP is a source of adenosine. J Biol Chem 284:33097-33106.

Kazmers IS, Mitchell BS, Dadonna PE, Wotring LL, Townsend LB, and Kelley WN (1981) Inhibition of purine nucleoside phosphorylase by 8-aminoguanosine: selective toxicity for T lymphoblasts. Science 214:1137-1139.

Lam PM, Mistry V, Marczylo TH, Konje JC, Evans MD, and Cooke MS (2012) Rapid measurement of 8-oxo-7,8-dihydro-2'-deoxyguanosine in human biological matrices using ultra-high-performance liquid chromatography-tandem mass spectrometry. Free Radic Biol Med 52:2057-2063.

Ohshima H, Sawa T, and Akaike T (2006) 8-Nitroguanine, a product of nitrative DNA damage caused by reactive nitrogen species: formation, occurrence, and implications in inflammation and carcinogenesis. Antioxid Redox Signal 8:1033-1045.

Osborne WR and Barton RW (1986) A rat model of purine nucleoside phosphorylase deficiency. Immunology 59:63-67.

Park EM, Shigenaga MK, Degan P, Korn TS, Kitzler JW, Wehr CM, Kolachana P, and Ames BN (1992) Assay of excised oxidative DNA lesions: isolation of 8-oxoguanine and its nucleoside derivatives from biological fluids with a monoclonal antibody column. Proc Natl Acad Sci USA 89:3375-3379.

Sodum RS, Nie G, and Fiala ES (1993) 8-Aminoguanine: a base modification produced in rat liver nucleic acids by the hepatocarcinogen 2-nitropropane. Chem Res Toxicol 6:269-276.

Address correspondence to: Dr. Edwin K. Jackson, Department of Pharmacology and Chemical Biology, 100 Technology Drive, Room 514, University of Pittsburgh School of Medicine, Pittsburgh, PA 15219. E-mail: edj@pitt.edu 